RF or IF EMF and glioma or meningioma risk, and the majority of odds ratios (ORs) were $<1.0$. Some elevated ORs were observed in the highest exposure quantile $\left(90^{\text {th }}\right)$ for both RF electric fields and IF magnetic fields in the 1-4 year exposure time window for glioma, but were not statistically significant.

Conclusions Lifetime occupational RF and IF exposures based on our index were not associated with the risk of glioma or meningioma. Further work should include more exposed participants and examine alternative exposure or dose indices such as those incorporating thresholds on biological effects or combinations of static and RF magnetic fields.

\section{Oral Presentation}

\section{Cancer}

\section{RISK OF PROSTATE CANCER IN FIREFIGHTERS: A META- ANALYSIS}

\begin{abstract}
1,2 Jeavana Sritharan* ${ }^{*}{ }^{3}$ Marie-Elise Parent, ${ }^{1}$ Manisha Pahwa, ${ }^{1,4}$ Shelley Harris, ${ }^{4}$ Donald Cole, ${ }^{1,4}$ Paul Demers. ${ }^{1}$ Occupational Cancer Research Centre, Cancer Care Ontario, Toronto, Ontario, Canada; ${ }^{2}$ Institute of Medical Science, University of Toronto, Toronto, Ontario, Canada; ${ }^{3}$ INRS-Institut Armand-Frappier, University of Quebec, Laval, Quebec, Canada; ${ }^{4}$ Dalla Lana School of Public Health, University of Toronto, Toronto, Ontario, Canada
\end{abstract}

10.1136/oemed-2017-104636.299

Objectives Although epidemiological studies have generally shown inconsistent findings for prostate cancer risk and occupation, some associations with firefighting have been observed. Firefighters are exposed to harmful substances that include known carcinogens. This meta-analysis aimed to synthesise the available published evidence on prostate cancer risk in firefighters.

Methods A comprehensive search of PubMed and Web of Science was conducted for all epidemiological studies on firefighting and prostate cancer published in English from 19802017. Studies that reported on risk estimates for incidence and/or mortality with corresponding ever/never employment were extracted from included studies. Meta risk estimates (mRE) were determined in a random effects model and between-study heterogeneity was evaluated based on $\mathrm{I}^{2}$ values.

Results Of the 101 studies retrieved, 25 firefighter studies were included in this analysis. Ever employment as a firefighter, based on results from all studies, was associated with a $15 \%$ increase in prostate cancer risk (95\% CI 1.04-1.27, $\left.\mathrm{I}^{2}=71 \%\right)$. By study design, the mRE was 1.14 (95\% CI $1.03-$ $\left.1.26, \mathrm{I}^{2}=67 \%\right)$ for 18 cohort studies and 1.22 (95\% CI $0.90-$ $1.67, \mathrm{I}^{2}=82 \%$ ) for 5 case-control studies. Incidence studies had an mRE of 1.17 (95\% CI 1.07-1.28, I $\left.\mathrm{I}^{2}=74 \%\right)$ and mortality studies had an mRE of 1.12 (95\% CI $0.92-1.36$, $\left.\mathrm{I}^{2}=50 \%\right)$. For linkage and registry-based studies, an $\mathrm{mRE}$ of 1.19 (95\% CI 1.05-1.33, $\left.\mathrm{I}^{2}=61 \%\right)$ was observed. Estimates showed moderate to substantial heterogeneity.

Conclusions This analysis showed evidence of a small excess risk of prostate cancer in firefighters across study designs. Further evaluation of heterogeneity, potential biases, and quality assessment are currently underway.

\section{Poster Presentation}

\section{Burden of Disease}

\section{ESTIMATION OF THE BURDEN OF CHRONIC OBSTRUCTIVE PULMONARY DISEASE DUE TO OCCUPATION IN GREAT BRITAIN}

${ }^{1}$ Sally Hutchings*, ${ }^{1}$ Lesley Rushton, ${ }^{2}$ Steven Sadhra, ${ }^{3,4}$ David Fishwick. ${ }^{1}$ Imperial College London, Department of Epidemiology and Biostatistics, London, UK; ${ }^{2}$ Occupational and Environmental Medicine, College of Medical and Dental Sciences, University of Birmingham, Birmingham, UK; ${ }^{3}$ Health and Safety Laboratory, Buxton, UK; ${ }^{4}$ Infection, Immunity and Cardiovascular Disease, University of Sheffield, Sheffield, UK

\subsection{6/oemed-2017-104636.300}

Introduction We have estimated the burden of COPD for deaths and prevalence due to occupational exposures in Britain using published literature and available national data plus a population based job exposure matrix (ACEJEM). The prevalence estimate avoids underestimation of the burden as estimates of COPD deaths, or hospitalisations, only account for the most serious cases.

Methods We used population attributable fractions (PAFs), requiring estimates of the population exposed to airborne pollutants at work, derived from 2001 Census data by job code matched to a JEM giving proportions exposed and average levels of exposure to pollutants including vapours, gases, dusts, fumes (VGDF) by UK SOC2000 job code. Exposures were matched to the best currently available population-based risk estimates. We estimated prevalent COPD PAFs using a novel age-time window approach to estimate prevalent numbers exposed, and deaths from COPD which requires numbers ever exposed were estimated using a risk exposure period methodology, taking into account age.

Results The PAF for prevalent COPD due to occupational exposure to VGDF was $18.7 \%$ for men, $8.0 \%$ for women and $11.5 \%$ overall giving 22700 cases in men and 13400 in women. Equivalent PAFs for COPD deaths, based on exposure $30+$ years before 2010, were 18.3\%/6.1\%/12.3\% (2,480 deaths in men, 790 in women). Prevalent PAF estimates which included a 30 year onset lag and mortality PAFs assuming 080 year latencies produced very similar results.

Conclusions The novel approach, making best use of available data, provides burden estimates which may inform strategies for reducing workplace COPD risks.

\section{Oral Presentation}

\section{Burden of Disease}

\section{6 COMPARING ALTERNATIVE METHODS OF ESTIMATING FUTURE DISEASE BURDEN DUE TO OCCUPATION}

${ }^{1}$ Sally Hutchings*, 'Lesley Rushton, ${ }^{2}$ Martie van Tongeren, ${ }^{3}$ Damien McElvenny. ${ }^{1}$ Imperial College London, Department of Epidemiology and Biostatistics, London, UK; ${ }^{2}$ University of Manchester, Manchester, UK; ${ }^{3}$ Institute of Occupational Medicine, Edinburgh, UK

10.1136/oemed-2017-104636.301 